\title{
ERRATUM
}

\section{HIGH ORDER RANDOM WALKS: BEYOND SPECTRAL GAP}

\author{
TALI KAUFMAN*, IZHAR OPPENHEIM**
}

Received January 1, 2021

Revised August 11, 2021

This is an erratum to [1, Theorem 1.5 (3), Theorem 5.10, Corollary 5.11].

We recall that for a two-sided $\lambda$-local spectral expander, we defined constants:

$$
\varepsilon_{k}= \begin{cases}\lambda & k=0 \\ 2 k(1+2 k \sqrt{k}) \varepsilon_{k-1}+(k+1) \lambda & 0<k\end{cases}
$$

(see $[1$, Theorem 5.6]). With this definition, the corrected version of $[1$, Theorem 5.10] is:

Theorem 1. Let $X$ is a two-sided $\lambda$-local spectral expander, and let $\left\{\varepsilon_{k}: 0 \leq k \leq n-1\right\}$ be the constants defined above. Assume that $\varepsilon_{k} \leq \frac{1}{2(1+2(k+1) \sqrt{k+1})}$ for all $0 \leq k \leq n-2$ and $\varepsilon_{n-1}<\frac{1}{2 \sqrt{n}}$. For every $0 \leq k \leq n-1$ and $0 \leq j_{0} \leq k$, if $\varphi \in U_{k}^{j_{0}}$, then

$$
\left\|\varphi-P_{W_{k}^{j_{0}}} \varphi\right\| \leq \sqrt{k+2} \varepsilon_{k}\|\varphi\| .
$$

Moreover, for every $\phi \in C_{0}^{k}(X)$,

$$
\begin{aligned}
& \left\|P_{W_{k}^{j}} \phi\right\|^{2} \leq\left\|P_{U_{k}^{j}} \phi\right\|^{2}+\left(\sqrt{k+2} \varepsilon_{k}+\frac{\sqrt{k+1} \varepsilon_{k}}{1-\sqrt{k+1} \varepsilon_{k}}\right)\|\phi\|^{2}, \\
& \left\|P_{W_{k}^{j}} \phi\right\|^{2} \geq\left\|P_{U_{k}^{j}} \phi\right\|^{2}-\left(\sqrt{k+2} \varepsilon_{k}+\frac{\sqrt{k+1} \varepsilon_{k}}{1-\sqrt{k+1} \varepsilon_{k}}\right)\|\phi\|^{2} .
\end{aligned}
$$

Mathematics Subject Classification (2010): 05E45, 05C81

* Research supported by ERC and BSF.

** Research supported by ISF. 
Proof. The proof of the inequality

$$
\left\|\varphi-P_{W_{k}^{j_{0}}} \varphi\right\| \leq \sqrt{k+2} \varepsilon_{k}\|\varphi\|, \forall \varphi \in U_{k}^{j_{0}}
$$

given in [1] is correct and thus we are left to prove the second and third inequalities. Fix $0 \leq j \leq k$. To avoid cumbersome notation, we denote $U=$ $U_{k}^{j}, W=W_{k}^{j}$. Note that by (1), it holds that

$$
\left\|\left(I-P_{W}\right) P_{U}\right\| \leq \sqrt{k+2} \varepsilon_{k},
$$

and thus

$$
\left\|P_{U}\left(I-P_{W}\right)\right\| \leq \sqrt{k+2} \varepsilon_{k} .
$$

By [1, Theorem 5.9], it holds that

$$
\left\|\left(I-P_{U}\right) P_{W} \phi\right\| \leq \frac{\sqrt{k+1} \varepsilon_{k}}{1-\sqrt{k+1} \varepsilon_{k}}\left\|P_{W} \phi\right\|,
$$

and thus

$$
\left\|P_{W}\left(I-P_{U}\right)\right\|=\left\|\left(I-P_{U}\right) P_{W}\right\| \leq \frac{\sqrt{k+1} \varepsilon_{k}}{1-\sqrt{k+1} \varepsilon_{k}} .
$$

We note that

$$
\begin{gathered}
\left\|P_{W} \phi\right\|^{2}=\left\langle P_{W} \phi, \phi\right\rangle= \\
\left\langle P_{W} \phi, P_{U} \phi\right\rangle+\left\langle P_{W} \phi,\left(I-P_{U}\right) \phi\right\rangle= \\
\left\|P_{U} \phi\right\|^{2}+\left\langle\left(P_{W}-P_{U}\right) \phi, P_{U} \phi\right\rangle+\left\langle\left(I-P_{U}\right) P_{W} \phi, \phi\right\rangle= \\
\left\|P_{U} \phi\right\|^{2}+\left\langle P_{U}\left(P_{W}-I\right) \phi, \phi\right\rangle+\left\langle\left(I-P_{U}\right) P_{W} \phi, \phi\right\rangle .
\end{gathered}
$$

It follows that

$$
\begin{aligned}
\left\|P_{W} \phi\right\|^{2} & \leq\left\|P_{U} \phi\right\|^{2}+\left|\left\langle P_{U}\left(I-P_{W}\right) \phi, \phi\right\rangle\right|+\left|\left\langle\left(I-P_{U}\right) P_{W} \phi, \phi\right\rangle\right| \\
& \leq\left\|P_{U} \phi\right\|^{2}+\left(\left\|P_{U}\left(I-P_{W}\right)\right\|+\left\|\left(I-P_{U}\right) P_{W}\right\|\right)\|\phi\|^{2} \\
& \stackrel{(2),(3)}{\leq}\left\|P_{U} \phi\right\|^{2}+\left(\sqrt{k+2} \varepsilon_{k}+\frac{\sqrt{k+1} \varepsilon_{k}}{1-\sqrt{k+1} \varepsilon_{k}}\right)\|\phi\|^{2},
\end{aligned}
$$

as needed. Similarly,

$$
\left\|P_{W} \phi\right\|^{2} \geq\left\|P_{U} \phi\right\|^{2}-\left(\sqrt{k+2} \varepsilon_{k}+\frac{\sqrt{k+1} \varepsilon_{k}}{1-\sqrt{k+1} \varepsilon_{k}}\right)\|\phi\|^{2} .
$$

Next, we prove a corrected version of Corollary [1, Corollary 5.11]: 
Corollary 2. Let $X$ is a two-sided $\lambda$-local spectral expander, and let $\left\{\varepsilon_{k}: 0 \leq k \leq n-1\right\}$ be the constants defined above. Assume that $\varepsilon_{k} \leq \frac{1}{2(1+2(k+1) \sqrt{k+1})}$ for all $0 \leq k \leq n-2$ and $\varepsilon_{n-1}<\frac{1}{2 \sqrt{n}}$. For $0 \leq k \leq n-1$ and $i \in \mathbb{N}$, define

$$
b_{k}(i, \lambda)=\sum_{j=0}^{k}\left(\frac{k+1-j}{k+2}+\frac{\sqrt{k+1}}{k+2} \varepsilon_{k}\right)^{2 i}\left(\frac{\sqrt{k+1} \varepsilon_{k}}{1-\sqrt{k+1} \varepsilon_{k}}\right) .
$$

Then for every $0 \leq k \leq n-1$, every $i \in \mathbb{N}$ and every $\phi \in C_{0}^{k}(X)$,

$$
\left\|\left(M_{k}^{+}\right)^{i} \phi\right\| \leq \sqrt{\sum_{j=0}^{k}\left(\left(\frac{k+1-j}{k+2}+\frac{\sqrt{k+1}}{k+2} \varepsilon_{k}\right)^{2 i}+b_{k}(i, \lambda)\right)\left\|P_{U_{k}^{j}} \phi\right\|^{2}},
$$

and

$$
\left\|\left(M_{k}^{+}\right)^{i} \phi\right\| \geq \sqrt{\sum_{j=0}^{k}\left(\frac{k+1-j}{k+2}-\frac{\sqrt{k+1}}{k+2} \varepsilon_{k}\right)^{2 i}\left(1-b_{k}(i, \lambda)\right)\left\|P_{U_{k}^{j}} \phi\right\|^{2}} .
$$

Proof. The proofs of the two inequalities are similar and we will prove only the first one and leave the second one to the reader. Fix $0 \leq k \leq n-1$. Every $\phi \in C_{0}^{k}$ has two orthogonal decompositions:

$$
\phi=\sum_{j=0}^{k} P_{W_{k}^{j}} \phi
$$

and

$$
\phi=\sum_{j=0}^{k} P_{U_{k}^{j}} \phi
$$

By definition, $W_{k}^{j}$ is an invariant subspace spanned by eigenvectors with eigenvalues in $\left[\frac{k+1-j}{k+2}-\frac{\sqrt{k+1}}{k+2} \varepsilon_{k}, \frac{k+1-j}{k+2}+\frac{\sqrt{k+1}}{k+2} \varepsilon_{k}\right]$. Thus $M_{k}^{+} P_{W_{k}^{j}}=$ $P_{W_{k}^{j}} M_{k}^{+} P_{W_{k}^{j}}$ and $\left\|M_{k}^{+} P_{W_{k}^{j}}\right\| \leq \frac{k+1-j}{k+2}+\frac{\sqrt{k+1}}{k+2} \varepsilon_{k}$. Combining these facts yields

$$
\begin{aligned}
\left\|\left(M_{k}^{+}\right)^{i} \phi\right\|^{2} & =\sum_{j=0}^{k}\left\|P_{W_{k}^{j}}\left(M_{k}^{+}\right)^{i} \phi\right\|^{2}=\sum_{j=0}^{k}\left\|\left(P_{W_{k}^{j}} M_{k}^{+}\right)^{i} P_{W_{k}^{j}} \phi\right\|^{2} \\
& \leq \sum_{j=0}^{k}\left(\frac{k+1-j}{k+2}+\frac{\sqrt{k+1}}{k+2} \varepsilon_{k}\right)^{2 i}\left\|P_{W_{k}^{j}} \phi\right\|^{2} .
\end{aligned}
$$


By Theorem 1,

$$
\left\|P_{W_{k}^{j}} \phi\right\|^{2} \leq\left\|P_{U_{k}^{j}} \phi\right\|^{2}+\left(\sqrt{k+2} \varepsilon_{k}+\frac{\sqrt{k+1} \varepsilon_{k}}{1-\sqrt{k+1} \varepsilon_{k}}\right)\|\phi\|^{2},
$$

and it follows that

$$
\begin{aligned}
& \left\|\left(M_{k}^{+}\right)^{i} \phi\right\|^{2} \\
& \leq \sum_{j=0}^{k}\left(\frac{k+1-j}{k+2}+\frac{\sqrt{k+1}}{k+2} \varepsilon_{k}\right)^{2 i}\left(\left\|P_{U_{k}^{j}} \phi\right\|^{2}+\left(\frac{\sqrt{k+1} \varepsilon_{k}}{1-\sqrt{k+1} \varepsilon_{k}}\right)\|\phi\|^{2}\right) \\
& =\sum_{j=0}^{k}\left(\frac{k+1-j}{k+2}+\frac{\sqrt{k+1}}{k+2} \varepsilon_{k}\right)^{2 i}\left\|P_{U_{k}^{j}} \phi\right\|^{2} \\
& \quad+\sum_{j=0}^{k}\left(\frac{k+1-j}{k+2}+\frac{\sqrt{k+1}}{k+2} \varepsilon_{k}\right)^{2 i}\left(\frac{\sqrt{k+1} \varepsilon_{k}}{1-\sqrt{k+1} \varepsilon_{k}}\right)\|\phi\|^{2} \\
& =\sum_{j=0}^{k}\left(\frac{k+1-j}{k+2}+\frac{\sqrt{k+1}}{k+2} \varepsilon_{k}\right)^{2 i}\left\|P_{U_{k}^{j}} \phi\right\|^{2}+b_{k}(i, \lambda)\|\phi\|^{2} .
\end{aligned}
$$

Using the fact that

$$
\|\phi\|^{2}=\sum_{j=0}^{k}\left\|P_{U_{k}^{j}} \phi\right\|^{2},
$$

completes the proof.

Remark 3 (Mistakes in the original proof and false inequalities). The mistake in the original proof of [1, Theorem 5.10] was very silly: We showed that for every $j^{\prime} \neq j$ it followed that $\left\|P_{W_{k}^{j^{\prime}}} P_{U_{k}^{j}}\right\| \leq \sqrt{k+2} \varepsilon_{k}$ (which is correct), but falsely deduced from it that $\left\|P_{W_{k}^{j}} P_{U_{k}^{j^{\prime}}} \phi\right\| \leq \sqrt{k+2} \varepsilon_{k}\left\|P_{U_{k}^{j}} \phi\right\|$. The mistake came from a silly indexation mistake - we confused $P_{W_{k}^{j}} P_{U_{k}^{j^{\prime}}}$ with $P_{W_{k}^{j^{\prime}}} P_{U_{k}^{j}}$. We then got the following false inequalities: for every $\phi$

$$
\left\|P_{W_{k}^{j}} \phi\right\| \leq\left(1+(k+1) \sqrt{k+2} \varepsilon_{k}\right)\left\|P_{U_{k}^{j}} \phi\right\|
$$

and

$$
\left\|P_{W_{k}^{j}} \phi\right\| \geq\left(1-(k+1) \sqrt{k+2} \varepsilon_{k}\right)\left\|P_{U_{k}^{j}} \phi\right\| .
$$

We did not notice it when submitting the paper, but if these inequalities were correct they would imply that $U_{k}^{j}=W_{k}^{j}$. Indeed, let $\phi \perp U_{k}^{j}$, then by 
the first inequality it follows that $\phi \perp W_{k}^{j}$. Similarly, if $\phi \perp W_{k}^{j}$, then by the second inequality (for $\varepsilon_{k}$ sufficiently small) it follows that $\phi \perp U_{k}^{j}$. Thus, $\left(W_{k}^{j}\right)^{\perp}=\left(U_{k}^{j}\right)^{\perp}$ and $U_{k}^{j}=W_{k}^{j}$. However, we do not expect $U_{k}^{j}=W_{k}^{j}$ to hold in general, since $U_{k}^{j}$ are only approximations of the eigenspaces and not the actual eigenspaces.

Remark 4. Note that for every $j>0$,

$$
\lim _{i \rightarrow \infty} \frac{b_{k}(\lambda, i)}{\left(\frac{k+1-j}{k+2}+\frac{\sqrt{k+1}}{k+2} \varepsilon_{k}\right)^{2 i}}=\infty .
$$

This means that when preforming many steps of the random walk, the decomposition to $U_{k}^{i}$ spaces does not give a good approximation of the decomposition to eigenspaces. In other words, the fact that the spaces $U_{k}^{i}$ and $W_{k}^{i}$ are not identical (but only very close to one another) comes into play when preforming many steps. This behavior did not appear in the incorrect version of the Corollary in [1].

Theorem [1, Theorem 1.5 (3)] should also be corrected to the statement in Corollary 2.

\section{References}

[1] T. Kaufman and I. Oppenheim: High order random walks: beyond spectral gap, Combinatorica 40 (2020), 245-281.

Tali Kaufman

Department of Computer Science

Bar-Ilan University

kaufmant@mit.edu
Izhar Oppenheim

Department of Mathematics

Ben-Gurion University of the Negev

Be'er Sheva 84105, Israel

izharo@bgu.ac.il 